Cahiers $d u$ MONDE RUSSE

\section{Cahiers du monde russe}

Russie - Empire russe - Union soviétique et États indépendants

$50 / 2-3 \mid 2009$

L'Europe orientale, 1650-1730. Crises, conflits et renouveau

\title{
Defending Rights in RussiaPamela A. JORDAN
}

, Vancouver : UBC Press, 2005, 304 p.

\section{Aurore Chaigneau}

\section{(2) OpenEdition}

\section{Journals}

Édition électronique

URL : https://journals.openedition.org/monderusse/9779

DOI : $10.4000 /$ monderusse. 9779

ISSN : $1777-5388$

Éditeur

Éditions de l'EHESS

Édition imprimée

Date de publication : 15 septembre 2009

ISBN : 978-2-7132-2260-3

ISSN : $1252-6576$

Référence électronique

Aurore Chaigneau, "Defending Rights in RussiaPamela A. JORDAN », Cahiers du monde russe [En ligne], 50/2-3 | 2009, mis en ligne le 14 janvier 2013, consulté le 03 septembre 2022. URL : http:// journals.openedition.org/monderusse/9779; DOI : https://doi.org/10.4000/monderusse.9779

Ce document a été généré automatiquement le 3 septembre 2022

Tous droits réservés 


\section{Defending Rights in RussiaPamela A. JORDAN}

, Vancouver : UBC Press, 2005, 304 p.

Aurore Chaigneau

\section{Pamela A. JORDAN, Defending Rights in Russia, Lawyers, the State, and Legal Reform in the Post-Soviet Era, Vancouver : UBC Press, 2005, $304 \mathrm{p}$.}

1 L'étude de Pamela A. Jordan sur la profession d'avocat dans la Russie contemporaine constitue une illustration intéressante des travaux de sciences politiques nordaméricaines sur un groupe. À travers un angle chronologique puis thématique, le lecteur découvre la genèse d'une catégorie socioprofessionnelle nouvelle dans la Russie contemporaine (1980-2003). L'auteur a axé son analyse sur l'affirmation progressive des avocats vis-à-vis de la puissance publique. Elle traite de la dissociation des liens institutionnels entre les différentes structures soviétiques, de la formation de nouvelles entités parallèles, puis de leur consolidation dans des ligues et associations jusqu'à la réforme du statut du barreau (advokatura) en 2002. Les tractations des collèges d'avocats et les prises de position des différents leaders concernant les principales réformes sont méthodiquement documentées.

2 Par ricochet se dessine l'évolution de la place dévolue à l'avocat dans la procédure civile et pénale. Mais les enjeux internes à la profession et qui en constituent la spécificité participation à la phase préliminaire d'enquête, fonction de rassemblement des preuves, rôle d'assistance juridique, voire d'aide juridictionnelle gratuite et de défense des droits des citoyens - ne sont abordés qu'à travers des enjeux institutionnels autres. La pratique judiciaire de l'avocat n'est pas au centre de l'ouvrage : on en apprend très peu sur le rôle effectif de ce dernier dans la défense des droits, sur ses relations avec ses clients et avec les autres professionnels du droit (universitaires, magistrats, notaires ou forces de l'ordre), ou encore sur la façon dont il s'accommode du cadre légal dans lequel il exerce, celui-ci étant évoqué de façon formelle (salariat, obligation d'aide juridictionnelle, 
comptabilité des profits, fixation des honoraires, etc.). Tout ne saurait être traité dans un même ouvrage. Néanmoins, l'angle choisi par l'auteur - construction d'un espace institutionnel, plutôt qu'évolution de la fonction d'avocat -, impose quelques limites. Comment en effet traiter de l'indépendance d'une profession sans étudier ses pratiques?

3 De fait, l'intérêt légitime du lecteur pour une profession a pour corollaire inévitable une curiosité au moins aussi importante à l'égard de ses activités quotidiennes. L'auteur n'est hélas entré que très marginalement dans ces considérations, et ce malgré un panel important d'enquêtes effectuées dans les années 1990. Il en résulte ponctuellement des raccourcis excessifs quand il s'agit d'éclairer les réformes par l'opposition théorique entre droit de la Common Law et droit continental. L'ouvrage n'en est pas moins d'une grande rigueur empirique.

Des sept chapitres, nous retiendrons deux champs : la construction historique du barreau et ses relations intestines avec la puissance publique.

5 Le premier champ porte sur la généalogie du barreau. Les premiers barreaux sont institués sous Alexandre II à l'occasion des grandes réformes institutionnelles des années 1860. L'advokatura est une organisation emblématique de la constitution d'un État de droit, puisqu'elle constitue la première association professionnelle non étatique en Russie. Les premiers avocats vont ensuite se faire entendre rapidement par leurs prises de position publiques. Ils sont placés sous la tutelle du ministère de la Justice, qui a un pouvoir de révocation, et ne bénéficient alors pas d'un monopole de représentation. Malgré leurs pouvoirs peu étendus, le ministère va rapidement œuvrer à limiter leurs prérogatives en amont du procès ainsi que pendant son déroulement. La profession se politise promptement du fait de sa proximité avec les institutions étatiques et le pouvoir. Et nombreux seront les avocats à subir des sanctions pour leur manque d'allégeance à l'Empire - nombreux aussi, dès la fin du XIX ${ }^{e}$ siècle, à être enfermés et déportés.

6 À la révolution, les bolcheviks prennent le contrôle de l'advokatura. Le barreau est dissout par décret du 22 novembre 1917. Toute personne ayant une "conscience politique de droite » est interdite de plaidoirie. Dès 1917, le commissariat à la Justice promeut à leur place des collèges d'accusateurs et de défenseurs. On dit la profession décimée, notamment du fait de son inutilité dans le procès civil et pénal, cadencé par la recherche de la " vérité objective » laissée aux autres protagonistes. Les purges vont en outre largement renouveler ses membres. La profession subsiste malgré tout, mais marginalisée, déclassée et perçue avec défiance par le pouvoir, malgré sa mise au pas: $70 \%$ sont membres du parti. Néanmoins, les statistiques montrent qu'elle n'a pas totalement disparu (8 000 en 1930, 13000 dans les années 1970). La présence d'un avocat reste exceptionnellement de mise, marginalement, pour les procès criminels de mineurs et d'aliénés, et quelques autres cas. Elle est parfois demandée par les parties sans obligation. Les chiffres du ministère de la Justice indiquent qu'en proportion de la population du pays, le nombre d'avocats est globalement très faible.

7 Un changement de doctrine survient en 1957 avec Hruščev. Les avocats sont associés comme tous les juristes à la "construction de la légalité socialiste » et l'advokatura est dotée d'un statut en 1962. De cette évolution, il faut conclure non pas à une prise d'indépendance mais plutôt à un encadrement accru de la profession pour conforter et légitimer le pouvoir en place. Les avocats servent d'ailleurs de relais au ministère de la Justice en produisant des rapports annuels dans lesquels ils notent les «erreurs» observées lors des procès, ultime contribution au renforcement de la légalité! 
8 À partir de la perestroïka, on assiste à un changement de perspective et à une réelle émancipation de la profession. Toutefois, cet affranchissement ne se fait pas sans heurts. Les statuts de 1980 redéfinissaient les fonctions des avocats dans le procès mais, jusqu'en 1989, le parti garde un contrôle sur la nomination des avocats en limitant donc leur nombre de même que leurs activités. Néanmoins, des structures juridiques nouvelles vont permettre de développer des activités d'aide judiciaire partiellement en dehors du cadre traditionnel. En 1988, le ministère de la Justice assouplit les règles de fixation des honoraires dans le but de mettre fin à la pratique des appointements illégaux. La même année, le Soviet suprême institue le principe des coopératives et, l'année suivante, le ministère approuve la création de coopératives d'assistance juridique. Une nouvelle génération d'avocats, souvent anciens fonctionnaires de l'appareil judiciaire, s'engouffre dans la brèche. Ces coopératives se distinguent des bureaux de consultation par leur autonomie vis-à-vis des collèges ; elles ne sont pas soumises aux statuts de 1980 et leurs membres exemptés de l'obligation d'assistance juridictionnelle.

Parallèlement à l'augmentation des effectifs, la profession s'organise. Le premier congrès de l'Union des avocats se réunit à Moscou en février 1989. Il est concurrencé par l'Union des juristes. Puis est fondée en 1994 l'Union fédérale des avocats, regroupant les collèges traditionnels, face à laquelle se constitue, derrière Gasan Mirzoev, la Ligue des avocats de Russie, représentant les collèges indépendants. En dépit de leurs dissensions, la Ligue et l'Union fédérale créent en 2000 un organe unique, le Conseil fédéral de l'advokatura, pour négocier une future loi qui sera adoptée en 2002. Celle-ci réforme l'organisation territoriale des barreaux en créant une chambre "par sujet de la Fédération». Les avocats y sont affiliés individuellement et/ou collectivement (bureau, consultation, etc.), et sont chapeautés par une chambre fédérale. La loi institue des commissions régionales (kvalifikacionnaja kollegija), chargées de la nomination sur examen et de la surveillance disciplinaire des avocats. La chambre fédérale s'empresse d'adopter un code de déontologie rédigé par Vjačeslav Makarov et largement inspiré du code d'éthique prérévolutionnaire.

10 Le second champ étudie les amours et désamours avec le ministère. Plusieurs faits témoignent des relations intestines entretenues par les collèges d'avocats avec le ministère de la Justice dans la période intermédiaire (1988-2002). Sous la perestroïka, les avocats ont encore l'obligation de laisser leurs dossiers de consultation en libre accès. Ceux-ci sont étudiés par leurs collègues, qui rédigent un rapport annuel d'activité destiné au ministère de la Justice. Cette pratique, contraire au principe de confidentialité, est abandonnée en 1991.

11 Toujours dans cette période de transition, la libéralisation de l'activité d'avocat permet la constitution de nouvelles entités. Néanmoins, il faut toujours recueillir l'accord du ministère qui, seul, décide de la création de nouveaux collèges auxquels devront s'affilier ces coopératives nouvelles, puis, dans les années 1990, les entreprises juridiques privées. On remarque que la politique du ministère est très libérale jusqu'en 1994, date à laquelle un large transfert de fonctionnaires vers le secteur privé a déjà été opéré. Puis, en toute logique, la pression pour limiter l'accès à la profession se renforce (par l'arrêt des enregistrements, l'imposition de licences), en limitant la création de nouveaux bureaux et en faisant du lobbying de haut niveau pour éviter que les nouvelles réglementations (fiscales, sociales, etc.) ne nuisent trop au développement de ces activités juridiques indépendantes. Il est d'ailleurs regrettable que, relevant ce phénomène de transfuge, l'auteur n'en ait pas montré les implications et conséquences. L'historique des relations 
entre les différents acteurs et l'analyse de leurs rapports avec les juges et l'administration locale auraient permis de compléter les monographies de cabinets.

Ce phénomène est seulement évoqué comme un mouvement, alors qu'il s'agit d'une véritable privatisation de l'administration et de ce que nous appellerons une « compétence ». Il illustre l'idée selon laquelle le développement du droit dans le secteur privé se produit non pas ex nihilo mais par la transposition de pratiques et de moyens matériels et humains empruntés à l'ancienne administration (personnel, réseaux, mais aussi locaux). Ainsi, par exemple, le Mosjuriscentr [Centre des juristes de Moscou], dirigé par G. Mirzoev et créé en 1989 avec l'autorisation de l'administration locale et du parti, a débuté comme une agence gouvernementale de Moscou. Le chef du département de la Justice de Moscou l'a fortement soutenu et certains le considèrent comme une agence de la ville. Plusieurs cabinets sont d'ailleurs spécialisés dans des contentieux public/privé et leurs membres usent de leurs relations historiques avec les directions des administrations ou des entreprises locales.

13 L'étude de Jordan s'achève en 2003, soit un an après l'entrée en vigueur de la loi, et l'année même de l'adoption du Code d'éthique. Mais, déjà en 2003, l'auteur comprend que l'autonomisation progressive de cette activité a été rendue possible par la libéralisation des années 1990. L'instauration plus récente de procédures de contrôle (commission de qualification), l'adoption de règles encadrant les activités et les devoirs de l'avocat placent aujourd'hui la puissance publique dans un rôle nouveau de censeur qui inquiète la profession, soucieuse de garantir la pérennité de son activité. Ces cabinets et bureaux dirigés par d'anciens fonctionnaires semblent $a$ priori peu enclins à choisir l'affrontement direct avec l'administration. Pourtant l'affaire Ioukos et la tentative du ministère de faire rayer du barreau l'avocate responsable du dossier ont montré que l'antagonisme des intérêts faisait son œuvre. 